\title{
Machine detector interface studies: Layout and synchrotron radiation estimate in the future circular collider interaction region
}

\author{
Manuela Boscolo, ${ }^{1,}$ Helmut Burkhardt, ${ }^{2}$ and Michael Sullivan ${ }^{3}$ \\ ${ }^{1}$ INFN-LNF, Via E. Fermi 40, 00044 Frascati, Rome, Italy \\ ${ }^{2}$ CERN, CH-1211 Geneva 23, Switzerland \\ ${ }^{3}$ SLAC, Menlo Park, California 94025, USA \\ (Received 11 August 2016; published 27 January 2017)
}

\begin{abstract}
The interaction region layout for the $e^{+} e^{-}$future circular collider FCC-ee is presented together with a preliminary estimate of synchrotron radiation that affects this region. We describe in this paper the main guidelines of this design and the estimate of synchrotron radiation coming from the last bending magnets and from the final focus quadrupoles, with the software tools developed for this purpose. The design follows the asymmetric optics layout as far as incoming bend radiation is concerned with the maximum foreseen beam energy of $175 \mathrm{GeV}$ and we present a feasible initial layout with an indication of tolerable synchrotron radiation.
\end{abstract}

DOI: 10.1103/PhysRevAccelBeams.20.011008

\section{BEAM OPTICS DESIGN}

The future circular collider (FCC-ee) is a very challenging machine with unprecedented high $e^{+} e^{-}$beam energy, luminosity and circumference. This machine will work at different beam energies, from $45.6 \mathrm{GeV}$ up to $175 \mathrm{GeV}$, with a luminosity that goes from $2.1 \times 10^{36} \mathrm{~cm}^{-2} \mathrm{~s}^{-1}$ for the lower energy to $1.3 \times 10^{34} \mathrm{~cm}^{-2} \mathrm{~s}^{-1}$ for the highest one. The circumference is foreseen to be about $100 \mathrm{~km}$. The plan is to have a flexible interaction region design that allows for this large beam energy range. The crab-waist collision scheme has been chosen to reach the highest possible luminosity, so the crossing angle is $30 \mathrm{mrad}$. A parameter table for the FCC-ee collider at the top energy is reported in Table I. The design of the beam optics is described in Ref. [1], Ref. [2] and Ref. [3].

The large crossing angle together with the high beam energy may induce high synchrotron radiation (SR) in the interaction region and consequently into the detector. We can state that the SR in the interaction region drives the layout design. One of the most significant constraints is the requirement on the critical energy and power of the synchrotron radiation generated upstream of the interaction region (IR) that may shine into the detector. An additional constraint of the FCC-ee layout is the compatibility with the hadron collider FCC-hh, which drives the infrastructure design. In order to combine the two requests of a large crossing angle and the need to prevent high energy SR fans from going into the interaction point (IP), the IR optics have been designed asymmetrically so that the incoming beam from both sides

"manuela.boscolo@lnf.infn.it

Published by the American Physical Society under the terms of the Creative Commons Attribution 3.0 License. Further distribution of this work must maintain attribution to the author(s) and the published article's title, journal citation, and DOI. comes from the inner ring and the outgoing beam exits to the outer ring. In this way the outgoing beams are more strongly bent than the incoming beams thereby lowering the SR energy from the incoming beams. The main guideline for the IR optics has been to make the SR backgrounds tolerable using experience from the LEP2 accelerator. There the highest local critical energy was $72 \mathrm{keV}$ and was located $260 \mathrm{~m}$ from the IP (Ref. [4]). Consequently, the optics designers have been asked to keep critical energies from bending magnets below $100 \mathrm{keV}$ and located at least $100 \mathrm{~m}$ from the IP. In addition, a request has been made to keep all critical energies from around the ring below $1 \mathrm{MeV}$ in order to minimize neutron production. Table II reports the radiation critical energy for the arc dipoles of existing and future colliders with very different beam energies. FCC-ee has the highest beam and critical energy.

The displacement of the FCC-ee IP with respect to the FCC-hh central trajectory is $9.4 \mathrm{~m}$ and the maximum displacement between the two machines is $11.9 \mathrm{~m}$ which is located about $400 \mathrm{~m}$ from the IP. The size of this displacement is inversely proportional to the critical energy of the last upstream bend magnet for a given crossing angle collision. The final focus magnets of each incoming and outgoing beam are separate elements. In Fig. 1, we show the cryostats for the focusing and defocusing quadrupoles each of which carry individual superconducting coils for each beam line. The defocusing and focusing quadrupoles of the final focus doublet are QC1 and QC2. For the top energy design, QC1 is $3.2 \mathrm{~m}$ long, QC2 is $2.5 \mathrm{~m}$ and their gradients are $97 \mathrm{~T} / \mathrm{m}$ and $61.5 \mathrm{~T} / \mathrm{m}$, respectively.

\section{INTERACTION REGION LAYOUT AND SYNCHROTRON RADIATION}

The interaction region is one of the key issues of a collider, it determines its success. For this reason it requires 
TABLE I. FCC-ee beam parameters most related to IR design relative to the top beam energy of $175 \mathrm{GeV}$ case.

\begin{tabular}{lc}
\hline circumference $(\mathrm{km})$ & 100 \\
crossing angle at IP $(\mathrm{mrad})$ & 30 \\
$L^{*}(\mathrm{~m})$ & 2.2 \\
Luminosity/IP $\left(10^{34} \mathrm{~cm}^{-2} \mathrm{~s}^{-1}\right)$ & 1.3 \\
$\beta_{x}^{*}(\mathrm{~m})$ & 1 \\
$\beta_{y}^{*}(\mathrm{~mm})$ & 2 \\
$\epsilon_{x}(\mathrm{~nm})$ & 1.3 \\
$\epsilon_{y}(\mathrm{pm})$ & 2.5 \\
$\sigma_{x}^{*}(\mu \mathrm{m})$ & 36 \\
$\sigma_{y}^{*}(\mathrm{~nm})$ & 71 \\
beam current $(\mathrm{mA})$ & 6.6 \\
particles/bunch & $1.71 \times 10^{11}$ \\
bunch number & 81 \\
Total SR power $(\mathrm{MW})$ & 98.6 \\
SR energy loss/turn $(\mathrm{GeV})$ & 7.47 \\
Energy spread by SR \% & 0.141 \\
\hline \hline
\end{tabular}

a careful design, balancing the requirements from the accelerator and detector sides. The interaction region design has to provide high luminosity that can be used for physics studies in the detectors with tolerable backgrounds and radiation. In addition, the design has to include a luminosity monitor and masks to stop synchrotron radiation photons. The beam pipe dimensions have to be properly set according to the beam size and available space, with flexibility for possible future optics modifications.

Two independent approaches are used to study the synchrotron radiation in the IR. The program SYNC_BKG (originally made by Al Clark of LBNL) is used to trace the beam macroparticles through the magnets which have been sliced into 4 sections. It calculates the radiation critical energy and start and stop points of each fan from each magnet slice for each macroparticle. The program scans across the entire transverse profile of the beam and has the ability to include a non-Gaussian transverse beam-tail distribution to the nominal Gaussian beam distribution. The beam pipe and masks are modeled with elliptically shaped apertures (which can be circles) located at various z positions. The program originally could only study SR from final focus quads that were on axis but it has been enhanced to do offset and tilted quads and bend magnets. A new software Machine Detector interface

TABLE II. Critical energies from arc dipoles for different $e^{+} e^{-}$ colliders and the proton-proton collider FCC-hh.

\begin{tabular}{lcc}
\hline \hline Collider & $E_{\text {beam }}(\mathrm{GeV})$ & $E_{\text {critical }}(\mathrm{keV})$ \\
\hline DAФNE & 0.51 & 0.2 \\
LER Superkekb & 4 & 2 \\
LEP2 & 100 & 72 \\
FCC-ee & 175 & 1000 \\
FCC-hh & $50 \times 10^{3}$ & 5 \\
\hline \hline
\end{tabular}

Simulations has been created, and still developing, specifically for this purpose. MDISIM is a flexible tool that provides an evaluation of SR in the IR (Ref. [5]) and plots the SR fans in the IR. MDISIM combines the standard tools MAD-X [6], ROOT [7] and GEANT4 [8].

These two approaches have been used to evaluate the SR from bends as well as from final focus (FF) quads, to design the IR with beam pipe dimensions, to place masks and shieldings at proper locations. MDISIM is used here to evaluate SR from near and far bends, while modified version of SYNC_BKG is used to evaluate SR from FF quads and design the IR with masks and shieldings.

The layout of the interaction region is based on the symmetrical final focus scheme with $L^{*}=2.2 \mathrm{~m}$ (where $L^{*}$ is defined as the distance between the IP and the entrance of the first quadrupole) and asymmetric optics as far as incoming bend radiation is concerned (Ref. [1]); this optics is optimized for the maximum foreseen beam energy of $175 \mathrm{GeV}$. An expanded horizontal view of the FCC-ee IR layout $\pm 10 \mathrm{~m}$ from IP is shown in Fig. 1, in the picture the QC1 and QC2 final focus quadrupoles are shown in yellow and pink; SR masks placed between the quadrupoles are in blue and red. Also the free space defined by an angle of $100 \mathrm{mrad}$ needed for the detector is shown. Figure 2 represents a closer view of the IR $\pm 3 \mathrm{~m}$ from the IP. The beam pipe is circular from $\pm 0.5 \mathrm{~m}$ from the IP with a radius of $20 \mathrm{~mm}$. Inside the final focus quadrupoles the full horizontal physical aperture is $24 \mathrm{~mm}$ (12 mm radius). This physical aperture corresponds to $20 \sigma_{x}$ in the horizontal plane at the back end of the focusing quadrupole QC2. In the vertical plane $60 \sigma_{y}$ corresponds to $5 \mathrm{~mm}$ in the middle of the defocusing quadrupole QC1. We presently define the beam-stay-clear (BSC) to be $20 \sigma_{x}$ and $60 \sigma_{y}$. The beam pipe under the final focus magnets is presently considered

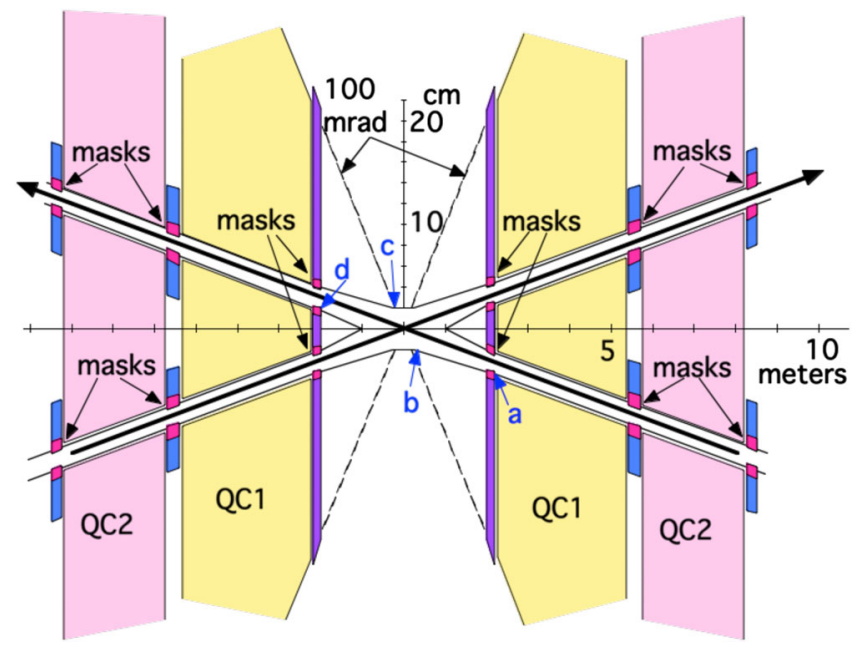

FIG. 1. View of the FCC-ee IR layout $\pm 10 \mathrm{~m}$ from IP. The QC1 and QC2 final focus quadrupoles are drawn in yellow and pink; the $a, b, c, d$ locations are defined. Between QC1 and QC2 there are SR masks (blue and red). 


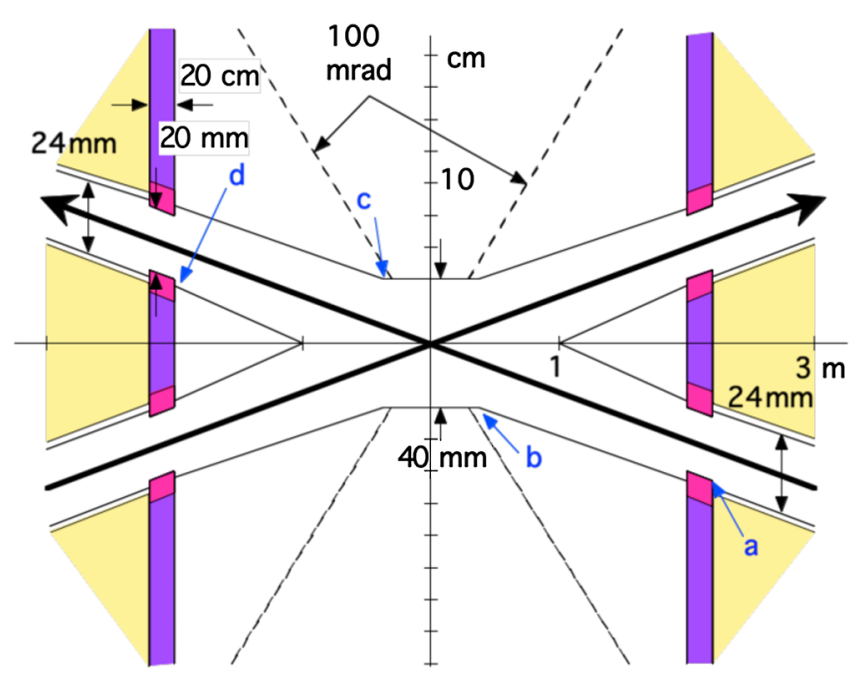

FIG. 2. Closer view of the FCC-ee IR layout $\pm 3 \mathrm{~m}$ from IP. In front of QC1 (in violet) is the possible location to the luminometer.

to be circular with circular masks. The SR masks have been placed in this layout close to these magnets, both before and after, reducing the physical aperture to $10 \mathrm{~mm}$ radius (see masks in Fig. 1 and Fig. 2). This ensures that the SR coming from the upstream bend magnet does not strike the cryogenic beam pipes.

In Fig. 1 and Fig. 2 the locations $a, b, c, d$ have been selected for detailed studies of the number and energy of the SR photons hitting these surfaces obtained with a modified version of SYNC_BKG. Positions $a$ and $b$ are upstream the IP, $c$ and $d$ downstream; $a$ corresponds to the back end of QC1, close to the beam pipe. $b$ is the upstream border where the pipe connects to the IP center beam pipe (20 mm radius). Similarly, $c$ is the downstream border in the upper corner where the IP center beam pipe connects to the beam pipe and $d$ is in the upfront position at the QC1 entrance. This study has the last upstream bend magnet fan sweeping in from the bottom of Fig. 1. Consequently, the bend fan strikes the bottom of the upstream mask surface $a$.

We want to establish a minimum beam pipe radius that does not intercept too many direct hit photons from upstream sources. Initially trying a $10 \mathrm{~mm}$ radius beam pipe revealed that radiation from both the final focus quads as well as the last upstream bend magnet would directly hit the central chamber with unacceptably high hit rates per beam bunch crossing. In addition, we found that the energy spectrum of the SR coming from the final focus (FF) quads was quite high with $50 \%>1 \mathrm{MeV}$ and $10 \%>10 \mathrm{MeV}$. There were about $8 \times 10^{5}$ photons hitting surfaces $b$ and $c$ from the FF quads and about $3 \times 10^{10}$ hitting these surfaces from the last upstream bend magnet. The bend magnet photon energy spectrum is a standard $100 \mathrm{keV}$ synchrotron photon energy distribution but the FF quadrupole radiation is significantly harder (see Fig. 3). We then proceeded to increase the central beam pipe radius and found that at a



FIG. 3. Plot of the photon energy spectra for the radiation coming from the quadrupoles and the upstream bend magnet and incident on the central chamber for a $10 \mathrm{~mm}$ radius pipe. The histogram starts at $1 \mathrm{MeV}$. The yellow histogram is all that is left of the $100 \mathrm{keV}$ critical energy radiation fan coming from the bend magnet. As one can see, the quadrupole radiation spectrum is significantly harder with some photons all the way out to $80 \mathrm{MeV}$ for each beam bunch crossing.

radius of $15 \mathrm{~mm}$ the central chamber just clears the synchrotron radiation sources and receives no direct hits. However, since the central chamber was partially shielding the downstream surface $d$ the rate on this surface increased. At this stage of the design, there have not been any parametric studies of orbit deviations or other factors that might change the exact location of the SR fans so the present decision is to make the central beam pipe radius $20 \mathrm{~mm}$ in order to have some margin in this respect. With the inner beam pipe radius established for now, we proceeded to look at scattered SR sources coming from local surfaces namely surfaces $a$ and $d$. From $a$ we find that about $2.5 \%$ of the incident photons will forward scatter toward the inner beam pipes and from surface $d$ about $0.5 \%$ will backscatter toward the inner beam pipes.

We can get a preliminary estimate of the photon rate on the central chamber from these secondary sources by calculating the solid angle fraction of the central beam pipe with respect to these scattering surfaces and assuming isotropic scattering. The energy spectrum of the scattered photons is used as a generating spectrum for photons incident on the central chamber at an average incident angle. This incident photon generator is fed to an EGS4 interface program to tally the number of incident photons on the central beam pipe that penetrate and hence are absorbed by the detector. In a similar manner we can get first estimates of the number of photons per beam bunch that are incident and then penetrate various beam pipe shield thicknesses in the study discussed below (see Sec. III). An additional note is that the above study was for symmetric FF optics with equal sized incoming and 
TABLE III. MDISIM output of the SR from the bending magnets upstream and downstream the IP. The first two columns show the element number and magnet name; followed by the end position of the magnet $s$ and magnet length $l$, the bending angles, critical energy, average number of photons radiated by the passage of a single electron, bending radius $\rho$, magnetic field, horizontal $\beta$ function, rms beam size and beam divergence, and the power radiated by SR in this magnet by a single beam, using the beam parameters of Table I.

\begin{tabular}{|c|c|c|c|c|c|c|c|c|c|c|c|c|}
\hline iele & name & $s \mathrm{~m}$ & $l \mathrm{~m}$ & $\begin{array}{l}\text { angle } \\
\text { mrad }\end{array}$ & $\begin{array}{c}E_{c} \\
\mathrm{keV}\end{array}$ & $n_{\gamma}$ & $\rho \mathrm{m}$ & $B \mathrm{~T}$ & $\beta_{x} \mathrm{~m}$ & $\begin{array}{c}\sigma_{x} \\
\mathrm{~mm}\end{array}$ & $\begin{array}{l}\text { divx } \\
\text { mrad }\end{array}$ & $\begin{array}{c}\text { Power } \\
\mathrm{kW}\end{array}$ \\
\hline \multicolumn{13}{|c|}{ upstream magnets, incoming beam: } \\
\hline 12 & BWL.2 & 155.1 & 55.08 & -0.4633 & 100. & 1.671 & 118906 & -0.0049 & 1868. & 1.5820 & 0.0099 & 0.3411 \\
\hline 16 & BC1L.2 & 268.4 & 109.2 & -0.9187 & 100 & 3.314 & 118886 & -0.0049 & 447.45 & 0.7743 & 0.0075 & 0.6764 \\
\hline 27 & BC2L.2 & 512.2 & 65.06 & -0.0499 & 9.117 & 0.18 & 1303954 & -0.0004 & 283.0 & 0.6158 & 0.0090 & 0.0033 \\
\hline 31 & BC3L.2 & 560.1 & 43.8 & -1.076 & 292.1 & 3.883 & 40694.5 & -0.0143 & 105.0 & 0.3750 & 0.0063 & 2.315 \\
\hline 35 & BC4L.2 & 608 & 43.8 & -1.653 & 448.7 & 5.963 & 26495.4 & -0.0220 & 288.4 & 0.6217 & 0.0063 & 5.462 \\
\hline 39 & BC5L.2 & 677.2 & 65.06 & -1.116 & 203.9 & 4.025 & 58313.8 & -0.0100 & 16.55 & 0.1489 & 0.0090 & 1.675 \\
\hline 56 & BL1.2 & 877.5 & 28.66 & 2.241 & 929.7 & 8.085 & 12787.8 & 0.0456 & 38.76 & 0.2279 & 0.0059 & 15.34 \\
\hline \multicolumn{13}{|c|}{ downstream magnets, outgoing beam: } \\
\hline 14 & BC1.1 & 65.24 & 38.04 & 2.211 & 691 & 7.976 & 17205.6 & 0.0339 & 57.05 & 0.2765 & 0.0080 & 11.25 \\
\hline 18 & BC2.1 & 76.69 & 7.352 & 0.4251 & 687.5 & 1.534 & 17293.6 & 0.0338 & 101.3 & 0.3684 & 0.0120 & 2.152 \\
\hline 22 & BC 3.1 & 109.7 & 28.96 & 3.586 & 1472 & 12.94 & 8073.9 & 0.0723 & 16.29 & 0.1477 & 0.0099 & 38.88 \\
\hline 29 & BC4.1 & 149.2 & 34.76 & 0.867 & 296.6 & 3.127 & 40088.5 & 0.0146 & 142.02 & 0.4362 & 0.0100 & 1.893 \\
\hline 33 & BC5.1 & 192.8 & 39.54 & 1.772 & 532.9 & 6.393 & 22308.1 & 0.0262 & 28.97 & 0.1970 & 0.0078 & 6.955 \\
\hline 37 & BC6.1 & 236.5 & 39.54 & 1.443 & 433.9 & 5.206 & 27396.4 & 0.0213 & 145.12 & 0.4410 & 0.0078 & 4.611 \\
\hline
\end{tabular}

outgoing beam pipes. We are also looking at an IR design with outgoing beam pipes that are larger in order to decrease the amount of trapped HOM power present in the IR. The only change to the above study for this other larger beam pipe case would be the loss of surface $d$ as a source of SR background as the radiation that did strike this surface now misses the entire downstream beam pipe.

Independent checks on SR from bending magnets have been performed with MDISIM. The optics used for these studies is v.74.11, corresponding to an asymmetrical design both for the bending magnets and the final focus interaction region as far as the last defocusing quadrupole is concerned. However the simulation studies discussed here refer only for the SR coming from the bends (Ref. [1]). MDISIM reads the machine lattice description from the MAD-X survey and twiss files and uses the information to generate a geometry file. The visualization of the geometry is done with EVE and OpenGL of ROOT. As a second step MDISIM performs an analytical estimate of the SR from bending magnets displaying for each element the main information related to SR, mainly critical and mean energy, radiated power, number of photons with the beam size and divergence and photon fan opening angle.

Table III shows the numerical estimates directly obtained by MDISIM for the bending magnets upstream of the IP (from $-877.5 \mathrm{~m}$ ) to downstream (up to $236.5 \mathrm{~m}$ ) of the IP.

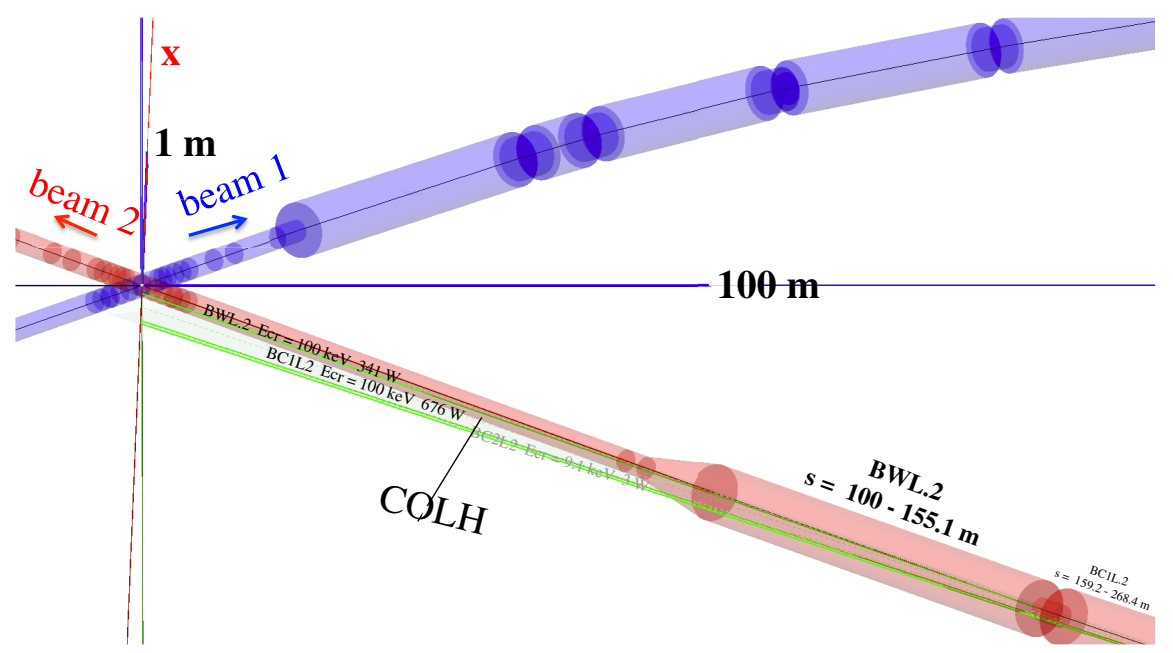

FIG. 4. SR from last bend upstream the IR from -155 to $-100 \mathrm{~m}$. Apertures are shown as circular with radius of $20 \mathrm{~mm}$ in the quads and $50 \mathrm{~mm}$ in the bends. The number of photons per crossing radiated by this bend towards the IP is $3.8 \times 10^{11}$ and the total energy is $1.2 \times 10^{7} \mathrm{GeV}$. 




FIG. 5. SR from bending magnets at about $600 \mathrm{~m}$ upstream the IR.

We note that for the outgoing beam the critical energy is above $100 \mathrm{keV}$ (sixth column in the lower rows) and the power emitted by all the bending magnets from IP to $250 \mathrm{~m}$ is $65.75 \mathrm{~kW}$. The incoming beam critical energy (sixth column in the upper rows) is $100 \mathrm{keV}$ from $-250 \mathrm{~m}$ to IP, going farther upstream the critical energy is higher. In addition the SR fans starting from $600 \mathrm{~m}$ upstream of the IP shine into the IP area, as appears in Fig. 5. Differently from LEP, here SR fans coming from far bends are directed to the IP.

The geometry is shown including the SR fans in Fig. 4 and Fig. 5 where in Fig. 4 are displayed the SR fans coming from $100 \mathrm{~m}$ and in Fig. 5 the ones from $677 \mathrm{~m}$, in both cases it is clear how they start upstream of the IP and go into the IP. In the two pictures there are also shown possible horizontal collimators, defined as $\mathrm{COLH}$, which might stop synchrotron photons which might shine onto the IP beam pipe.

As a last step detailed simulations of the passage of particles through materials can be performed directly using the MDISIM generated root geometry and importing the information to GEANT4, allowing GEANT4 to track the SR by generating synchrotron radiation photons. This last step is in progress.

\section{PHOTON COLLIMATION AND SHIELDING}

There are many different materials that can be used to collimate and shield photons upstream of the interaction region. Generally, these will be heavy materials like Tantalum (Ta, $Z=73$ ) or Tungsten ( $\mathrm{W}, Z=74)$. The case of lead is shown in Fig. 6. In Fig. 6 the green line at $10 \mathrm{keV}$ indicates that below this value photons in heavy material such as lead are not too dangerous, the red line at $100 \mathrm{keV}$ indicates it is hard to manage radiation above this value. Radiation around $10 \mathrm{MeV}$ would generate a continuous flux of neutrons generated by the giant dipole resonance and must therefore be avoided. Keep in mind that the synchrotron energy spectrum has a long high-energy tail for a given critical energy.

On the other hand, beam pipes are usually made of lighter materials like Aluminum $(\mathrm{Al}, Z=13)$ that is transparent for photons with energies higher than $40 \mathrm{keV}$ that also have small impact angles. Beryllium is also a very light material $(Z=3)$ and is transparent for photons with energy above $10 \mathrm{keV}$. To determine the proper material for the beam pipe, especially in the interaction region, it can be important to investigate the specular reflection at very small incident angles. All relevant cross sections for photon absorption and scattering are implemented in Geant4, with the exception of specular reflection of $\mathrm{keV}$ photons at very small scattering angles.

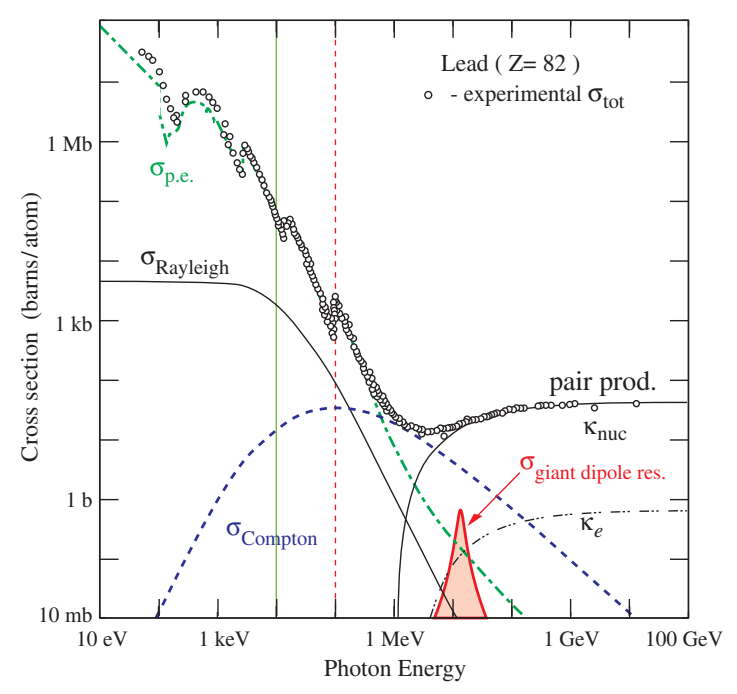

FIG. 6. Photon total cross section as a function of energy in lead showing the contribution of different processes. Green line is at $10 \mathrm{keV}$, red line is at $100 \mathrm{keV}$. Reference [9]. 


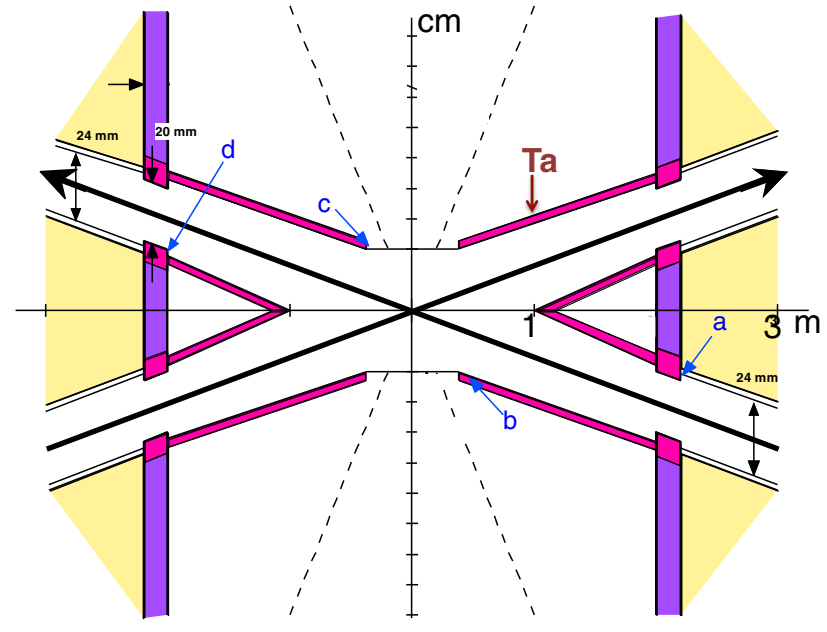

FIG. 7. Tantalum shielding is shown in red around the IR beam pipe from $0.5 \mathrm{~m}$ to $1.5 \mathrm{~m}$ from the IP.

A study on a Tantalum shield around the IR beam pipe from $0.5 \mathrm{~m}$ (the end of the central beam pipe) to $1.5 \mathrm{~m}$ from the IP has been started in order to study the rates of the photons through different shielding thicknesses. In Fig. 7 the Tantalum shielding around the beam pipe is shown in red. This part of the beam pipe does not receive any direct hits from upstream SR sources but a significant fraction of the photons hitting surfaces $a$ and $d$ either forward scatter or backscatter onto this intermediate beam pipe.

The method used for the study is analogous to the one described in Sec. II to design the IR. We generated the forward or backscattered energy spectrum of these photons and started them incident to the beam pipe surface with an average angle of incidence in order to estimate how many penetrated various thicknesses of shielding. The result is that the photon rate per bunch crossing is 727 through a $2 \mathrm{~mm}$ Ta shield, 1602 photons thru a $1 \mathrm{~mm}$ Ta shield and 3097 photons through a $0.5 \mathrm{~mm}$ Ta shield. These appear to be acceptable rates but we need to check this conclusion with a detailed detector design.

Detailed Geant 4 studies are also in progress on the detector side to estimate photon absorption and the impact of the remaining photon flux on detector performance.

\section{CONCLUSIONS}

In this paper we have focused on the interaction region layout with synchrotron radiation as a major design constraint. These studies are carried on within the FCCee Machine Detector Interface group, that has the goal of producing a feasible design of the interaction region in close collaboration with the optics group.

The next steps will be to define the masks and photon shielding in the interaction region, to perform beam and IP backgrounds evaluation with tracking into the interaction region and, if necessary, into the detector. There are studies ongoing of wake fields that may be generated in the IR region, and absorbers that will be needed for these electromagnetic fields as well as for the synchrotron radiation power.

The feasibility of the design has yet to be proven as there are several topics that we have not mentioned. For instance, a solenoid compensation scheme, magnet integration studies, final focus quadrupoles design, luminosity monitor and impedance budget estimate of the masks. In addition, the feasibility of the magnetic system as well as the underground infrastructure for the IR, not only for the challenge itself, but also for the constraint of requiring compatibility with the FCC-hh machine must also be studied more thoroughly. We see this present study as an initial encouraging step toward a robust IR design.

We would also like to mention that the compatibility of this design for the other running beam energies at 120, 90 and $45.6 \mathrm{GeV}$ has not been fully checked yet, and is planned as one of the next steps. The $46.5 \mathrm{GeV}$ operating point has had a very preliminary check and the design so far looks OK.

There are also ongoing studies to check the sustainability of the synchrotron radiation in the detector, and we need to determine if the LEP2 threshold value presently used can be relaxed a bit. These studies are carried on with dedicated Geant 4 studies and a fast and full simulation tool is in progress.

\section{ACKNOWLEDGMENTS}

The authors thank their colleagues for useful discussions and suggestions on FCC-ee accelerator and detector physics, mainly from the MDI and optics teams, and in particular N. Bacchetta, A. Blondel, A. Bogomyagkov, P. Janot, A. Kolano, M. Koratzinos, K. Oide, E. Perez, F. Zimmermann. This material is based upon work supported by the U.S. Department of Energy, Office of Science, Office of Basic Energy Sciences, under Contract No. DEAC02-76SF00515 and HEP.

[1] K. Oide et al., Design of beam optics for the future circular collider $e^{+} e^{-}$collider rings, Phys. Rev. Accel. Beams 19, 111005 (2016).

[2] K. Oide et al., Design of beam optics for the FCC-ee collider ring, in Proceedings, 7th International Particle Accelerator Conference (IPAC 2016) (JACoW, Geneva, 2016), p. THPOR022, http://accelconf.web.cern.ch/AccelConf/ ipac2016/papers/thpor022.pdf.

[3] A. Bogomyagkov, E. Levichev, and P. Piminov, Final focus designs for crab waist colliders, Phys. Rev. Accel. Beams 19, 121005 (2016).

[4] G. von Holtey et al., Study of beam induced particle backgrounds at the LEP detectors, Nucl. Instrum. Methods Phys. Res., Sect. A 403, 205 (1998). 
[5] H. Burkhardt and M. Boscolo, Tools for flexible optimisation of IR designs with application to FCC, in Proceedings, 6th International Particle Accelerator Conference (IPAC 2015) (JACoW, Geneva, 2015), p. 2072, https://jacowfs .jlab.org/conf/proceedings/IPAC2015/papers/tupty031.pdf.

[6] http://madx.web.cern.ch/madx/.
[7] https://root.cern.ch/.

[8] S. Agostinelli et al. (GEANT4 Collaboration), GEANT4: A simulation toolkit, Nucl. Instrum. Methods Phys. Res., Sect. A 506, 250 (2003).

[9] K. A. Olive et al. (Particle Data Group Collaboration), Review of particle physics, Chin. Phys. C 38, 090001 (2014). 